\title{
Winds of M- and S-type AGB stars: an unorthodox suggestion for the driving mechanism
}

\author{
S. Höfner ${ }^{1}$ and A. C. Andersen ${ }^{2}$ \\ 1 Department of Astronomy and Space Physics, Uppsala University, Box 515, 75120 Uppsala, Sweden \\ e-mail: hoefner@astro.uu.se \\ 2 Dark Cosmology Centre, Niels Bohr Institute, University of Copenhagen, Juliane Maries Vej 30, 2100 Copenhagen, Denmark \\ e-mail: anja@dark-cosmology.dk
}

Received 19 December 2006 / Accepted 15 February 2007

ABSTRACT

\begin{abstract}
Context. Current knowledge suggests that the dust-driven wind scenario provides a realistic framework for understanding mass loss from C-rich AGB stars. For M-type objects, however, recent detailed models demonstrate that radiation pressure on silicate grains is not sufficient to drive the observed winds, contrary to previous expectations.

Aims. In this paper, we suggest an alternative mechanism for the mass loss of M-type AGB stars, involving the formation of both carbon and silicate grains due to non-equilibrium effects, and we study the viability of this scenario.

Methods. We model the dynamical atmospheres and winds of AGB stars by solving the coupled system of frequency-dependent radiation hydrodynamics and time-dependent dust formation, using a parameterized description of non-equilibrium effects in the gas phase. This approach allows us to assess under which circumstances it is possible to drive winds with small amounts of carbon dust and to get silicate grains forming in these outflows at the same time.

Results. The properties of the resulting wind models, such as mass-loss rates and outflow velocities, are well within the observed limits for M-type AGB stars. Furthermore, according to our results, it is quite unlikely that significant amounts of silicate grains will condense in a wind driven by a force totally unrelated to dust formation, as the conditions in the upper atmosphere and wind acceleration region put strong constraints on grain growth.

Conclusions. The proposed scenario provides a natural explanation for the observed similarities in wind properties of M-type and C-type AGB stars and implies a smooth transition for stars with increasing carbon abundance, from solar-composition to C-rich AGB stars, possibly solving the longstanding problem of the driving mechanism for stars with a $\mathrm{C} / \mathrm{O}$ close to one.
\end{abstract}

Key words. stars: mass-loss - stars: atmosphere - hydrodynamics - stars: circumstellar matter - stars: AGB and post-AGB

\section{Introduction}

Low- and intermediate-mass stars are known to lose a significant fraction of their mass through slow, massive winds during the asymptotic giant branch (AGB) phase of their evolution. The basic scenario for this process is that of pulsation-enhanced dustdriven winds: shock waves created by stellar pulsation lead to a dense, cool, extended stellar atmosphere, allowing for efficient dust formation. The grains are accelerated away from the star by radiation pressure, dragging gas along. The composition of the grains is determined by the elemental abundances in the stellar atmosphere, with $\mathrm{C}$-rich stars forming carbon grains, and O-rich environments producing silicate particles.

This scenario seems to work well for the case of C-rich AGB stars, as demonstrated by comparison of detailed self-consistent dynamical models with observations as diverse as low-resolution IR spectra (e.g., Höfner et al. 2003; Gautschy-Loidl et al. 2004) and the line profile variations of $\mathrm{CO}$ vibration-rotation lines (e.g., Nowotny et al. 2005).

Recent work on similar models for stars with $\mathrm{C} / \mathrm{O}<1$, however, faces serious problems (Woitke 2006; Höfner 2007). Frequency-dependent dynamical models demonstrate that the opacities of silicate grains are too low to drive a wind, even when assuming fairly favorable grain compositions. In addition, these models show that the Fe-content of the grains has to be extremely low, as the radiative equilibrium temperature of silicate grains increases strongly with increasing inclusion of $\mathrm{Fe}$, which leads to even lower grain opacities than previously assumed. Therefore, the very ingredient that makes C-rich models more successful than ever, i.e. frequency-dependent radiative transfer, prevents dust-driven winds in stars with $\mathrm{C} / \mathrm{O}<1$.

While alternative wind mechanisms have been discussed earlier, observations indicate that winds of O-rich AGB stars behave very similarly to their C-rich counterparts. Furthermore, the very fact that silicate grains are formed in such outflows puts strict constraints on the possible mechanisms, as discussed in Sect. 3. This leads us to propose a scenario where the winds of M- (and probably S-) type AGB stars are actually driven by small amounts of carbon grains, with silicates forming as a byproduct. This requires a certain fraction of the carbon atoms in the dust formation region to be available for grain formation, i.e. not bound up in $\mathrm{CO}$.

This scenario implies deviations from chemical equilibrium $(\mathrm{CE})$ in the gas phase, probably brought about by strong atmospheric shock waves, which seems not too far-fetched in light of recent observations showing the existence of molecules typically expected in O-rich environments in C-rich stars (e.g., Schöier et al. 2006), and vice versa. Current models of nonequilibrium gas-phase chemistry by Cherchneff (2006) predict 
small deviations from CE (less than what we assume below), but these models are computed for significantly higher densities (which should favor more CE-like compositions) and without taking condensation into account. As an inclusion of detailed non-CE in the gas phase in our wind models is well beyond the scope of this first investigation, we use the abundance of available carbon as a parameter of the models. This approach allows us to test under which circumstances it is possible to drive winds and to get a significant amount of silicate grains forming in the outflows.

\section{The hydrodynamical model}

Our hydrodynamical models predict the mass-loss rates and wind velocities of AGB stars, as well as the amount of dust formed in the winds, by treating the atmosphere and the circumstellar environment around pulsating long-period variable stars in detail. This is done by solving the coupled system of frequency-dependent radiation hydrodynamics and timedependent dust formation (cf. Höfner et al. 2003). The calculations presented here are based on opacity sampling data of molecular opacities at 64 frequency points between 0.25 and $25 \mu \mathrm{m}$.

In contrast to our earlier models, we have implemented a time-dependent description of grain growth for silicate grains (Höfner et al., in prep.), and we focus on the formation of pure forsterite particles $\left(\mathrm{Mg}_{2} \mathrm{SiO}_{4}\right)$, as non-grey effects will force a low Fe-content (see Sect. 1). While treating the growth of grains in full non-equilibrium, we do not consider the nucleation of new grains from the gas phase but assume the presence of seed nuclei (a given number per $\mathrm{H}$ atom) at the point where grain growth becomes possible. Opacity data for the relevant grain materials is taken from Jäger et al. (2003; forsterite), Rouleau \& Martin (1991; amorphous carbon sample AC2), and Jäger et al. (1998; amorphous carbon sample cel400).

All models discussed here have solar elemental abundances (i.e. $\mathrm{C} / \mathrm{O} \approx 0.5$ ), but we assume that a certain fraction of carbon is not bound in $\mathrm{CO}$ in the region relevant for grain growth. This fraction is parameterized by a factor $F_{\mathrm{C}}=\tilde{\varepsilon}_{\mathrm{C}} / \varepsilon_{\mathrm{C}}$, where $\tilde{\varepsilon}_{\mathrm{C}}$ is the abundance of carbon available for dust formation. This is admittedly a very simple-minded treatment of non-equilibrium in the gas phase, but it allows us to assess how much free carbon is needed to drive a wind for given stellar parameters. Note also that only a fraction of this fraction of all carbon atoms will actually condense into grains (see Table 1 and discussion below).

\section{Results and discussion}

We have calculated a number of models with different combinations of stellar parameters, fractions of free carbon $\left(F_{\mathrm{C}}\right)$, and pulsation amplitudes, using two different sets of opacity data for amorphous carbon ${ }^{1}$. The model parameters and the resulting wind properties are summarized in Table 1, with symbols defined as follows:

- Model parameters and input data: luminosity $L_{\star}$; effective temperature $T_{\star}$ (initial model; mass $M_{\star}=1 M_{\odot}$ for all models); fraction of free carbon $F_{\mathrm{C}}$; period $P$ and velocity amplitude of piston $\Delta u_{\mathrm{p}}$ (luminosity factor $f_{\mathrm{L}}=2.0$ for all models, see Gautschy-Loidl et al. 2004); opacity data for amorphous

1 This term actually covers a variety of materials with different microscopic structures and different optical properties (see discussion in Andersen et al. (2003). carbon $\kappa_{\mathrm{C}}: \mathrm{RM}=$ data from Rouleau \& Martin (1991, sample AC2), J4 = data from Jäger et al. (1998, sample cel400).

- Resulting wind properties: mass loss rate $\dot{M}$, mean velocity at the outer boundary $\langle u\rangle$, degree of condensation of silicon $f_{\mathrm{Si}}$, of free carbon $\tilde{f}_{\mathrm{C}}$ and of all carbon $f_{\mathrm{C}}^{\text {tot }}$.

As expected, the fraction of free carbon required to obtain a dustdriven wind decreases with increasing luminosity and decreasing effective temperature. Also, in accordance with previous results on C-rich wind models, the mean degree of condensation of free carbon $\tilde{f}_{\mathrm{C}}$ is never close to one in these models, and the degree of condensation of all carbon (obtained by multiplying $\tilde{f}_{\mathrm{C}}$ with $\left.F_{\mathrm{C}}\right)$ is rather low in certain cases.

What may seem more surprising at first glance is that the degree of condensation for $\mathrm{Si}$ (and, correspondingly, the amount of silicate grains formed in the wind) is quite low in most models, and actually decreases with conditions that make the formation of carbon grains more efficient (e.g., higher pulsation amplitude, or a larger fraction of free carbon, for given stellar parameters). The low silicate dust abundance is not a matter of temperatures being too high to allow for condensation, but rather a matter of grain growth timescales: as soon as enough carbon dust is formed to accelerate the gas away from the star, the condensation of dust (and in particular of silicate grains, handicapped by lower abundances of critical elements like $\mathrm{Si}$ and $\mathrm{Mg}$ ) becomes a race against falling densities in the outflow, a phenomenon that finally stops condensation, both for silicates and carbon grains (leading to $\tilde{f}_{\mathrm{C}}<1$ ), despite falling gas and grain temperatures.

Therefore it is actually quite remarkable that silicate grains can form at all in a wind driven by some other force (e.g. radiation pressure on another, unrelated dust species as in this case), as the rapid slowing down of grain growth in an outflow puts tight constraints on the wind mechanism. If the silicate grains do not form simultaneously with (or maybe even prior to) the driving dust species (carbon), i.e. at the same distance from (or maybe even closer to) the star, at rather high gas densities, there is virtually no chance of their forming at all.

In order to understand how grains consisting of such different materials as amorphous carbon and silicates may form at basically the same distance from the star, we have to take a look at two types of temperatures: the condensation temperature of a material (setting the threshold for grain formation) and the grain temperature at a given distance from the star, which, in practice, will be equal to the radiative equilibrium temperature corresponding to the grain material. At the densities prevailing in the pulsating atmosphere, amorphous carbon grains will typically start to form around 1500-1800 K, while the formation of forsterite requires temperatures as low as $1000-1200 \mathrm{~K}$. However, due to their optical properties (opacity decreasing with wavelength around stellar flux maximum), carbon grains will be hotter than the black body temperature at a given distance (cf. Höfner et al. 2003, Fig. 6), while forsterite grains will be cooler (opacity increasing with wavelength in the critical regime) ${ }^{2}$. This means that, at the same distance from the star, forsterite grains will be significantly cooler than amorphous carbon grains, and the critical condensation temperature is reached approximately in the same region for both types of dust (Fig. 1).

\footnotetext{
2 At the relevant wavelengths, the opacity of amorphous carbon grains is roughly proportional to $1 / \lambda$. Therefore the carbon grains absorb and emit radiation preferentially at short wavelengths. This property, in combination with the radiative equilibrium condition, requires the grains to be hotter than the radiation temperature in order to emit as much energy as they absorb.
} 
Table 1. Model parameters $\left(L_{\star}, T_{\star}, F_{\mathrm{C}}, P, \Delta u_{\mathrm{p}}\right)$, dust opacity data $\left(\kappa_{\mathrm{C}}\right)$, and resulting wind properties $\left(\dot{M},\langle u\rangle, f_{\mathrm{Si}}, \tilde{f}_{\mathrm{C}}, f_{\mathrm{C}}^{\text {tot }}\right)$.

\begin{tabular}{llllllccccc}
\hline \hline$L_{\star}\left[L_{\odot}\right]$ & $T_{\star}[\mathrm{K}]$ & $F_{\mathrm{C}}$ & $\kappa_{\mathrm{C}}$ & $P[\mathrm{~d}]$ & $\Delta u_{\mathrm{p}}\left[\mathrm{km} \mathrm{s}^{-1}\right]$ & $\dot{M}\left[M_{\odot} / \mathrm{yr}\right]$ & $\langle u\rangle\left[\mathrm{km} \mathrm{s}^{-1}\right]$ & $f_{\mathrm{Si}}$ & $\tilde{f}_{\mathrm{C}}$ & $f_{\mathrm{C}}^{\text {tot }}$ \\
\hline 5000 & 2800 & 0.7 & $\mathrm{RM}$ & 310 & 6.0 & $5 \times 10^{-7}$ & 4 & $3 \times 10^{-2}$ & 0.3 & 0.2 \\
7000 & 2700 & 0.4 & $\mathrm{RM}$ & 390 & 6.0 & $2 \times 10^{-6}$ & 4 & $5 \times 10^{-2}$ & 0.4 & 0.2 \\
7000 & 2700 & 0.5 & $\mathrm{RM}$ & 390 & 6.0 & $3 \times 10^{-6}$ & 7 & $1 \times 10^{-2}$ & 0.4 & 0.2 \\
10000 & 2600 & 0.3 & $\mathrm{RM}$ & 525 & 6.0 & $1 \times 10^{-5}$ & 6 & $4 \times 10^{-2}$ & 0.5 & 0.2 \\
10000 & 2600 & 0.3 & $\mathrm{RM}$ & 525 & 3.0 & $5 \times 10^{-6}$ & 6 & $2 \times 10^{-1}$ & 0.5 & 0.2 \\
10000 & 2600 & 0.4 & $\mathrm{RM}$ & 525 & 3.0 & $7 \times 10^{-6}$ & 10 & $2 \times 10^{-2}$ & 0.4 & 0.2 \\
\hline 7000 & 2700 & 0.4 & $\mathrm{~J} 4$ & 390 & 6.0 & $1 \times 10^{-6}$ & 4 & $3 \times 10^{-1}$ & 0.3 & 0.1 \\
10000 & 2600 & 0.3 & $\mathrm{~J} 4$ & 525 & 3.0 & $4 \times 10^{-6}$ & 6 & $5 \times 10^{-1}$ & 0.4 & 0.1 \\
10000 & 2600 & 0.4 & $\mathrm{~J} 4$ & 525 & 3.0 & $6 \times 10^{-6}$ & 10 & $6 \times 10^{-2}$ & 0.4 & 0.2 \\
\hline
\end{tabular}
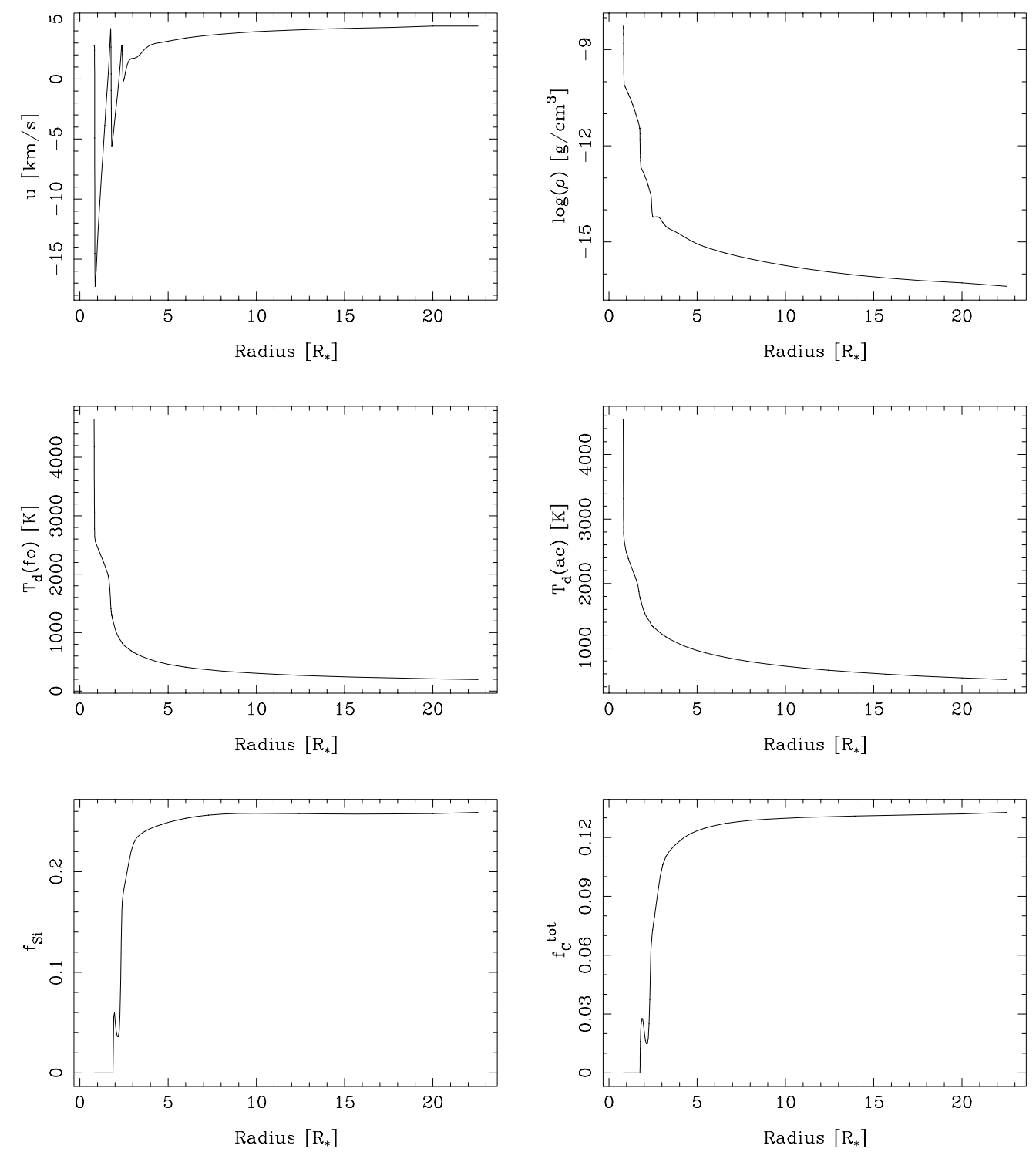

Fig. 1. Snapshot of the radial structure of the model with $L_{\star}=7000 L_{\odot}, T_{\star}=2700 \mathrm{~K}$ and opacity data for amorphous carbon by Jäger et al. (1998, sample cel400). Top row: gas velocity and density; middle row: radiative equilibrium temperature for forsterite and carbon grains; bottom row: degree of condensation for $\mathrm{Si}$ and $\mathrm{C}$. Radius in units of the stellar radius.

The strong sensitivity of a non-driving dust species (i.e. silicate grains in the present case) to the conditions in the wind acceleration region is illustrated by a comparison of models calculated with two different opacities for the carbon grains, but otherwise identical parameters. The data of Jäger et al. (1998, cel400) has a steeper slope in the region around $1 \mu \mathrm{m}$ than many other types of amorphous carbon (cf. Fig. 2 in Andersen et al. 2003), leading to a higher grain temperature at a given distance. Therefore, the formation of carbon grains (and, consequently the wind acceleration region) is shifted slightly outwards in these models compared to the ones based on the Rouleau \& Martin (1991) data, improving the conditions for the formation of 
silicate grains. This results in an increased degree of condensation for $\mathrm{Si}$, while the mass-loss rate and wind velocity (governed by the carbon grains) are basically unchanged (cf. Table 1$)^{3}$. These results indicate that it may be very difficult to form silicate grains in a wind driven by a force totally unrelated to dust formation, as the conditions in the upper atmosphere and wind acceleration region put strong constraints on grain growth.

In addition to these theoretical considerations, several existing observations can be interpreted in favor of the proposed scenario: Schöier et al. (2006) have recently demonstrated the existence of molecules typical of O-rich environments in C-rich AGB stars, a finding that is hard to reconcile with standard chemical equilibrium models. Interferometric and spectroscopic observations indicate the existence of very dense layers of water vapor in O-rich stars (e.g., Weiner 2004; Ohnaka 2005; Justtanont 2005), potentially requiring abundances of $\mathrm{H}_{2} \mathrm{O}$ in excess of what can be expected from chemical equilibrium (where an amount of oxygen equal to the total amount of carbon is bound in $\mathrm{CO}$ ). Furthermore, while the wind properties of $\mathrm{C}$-rich and O-rich AGB stars are very similar, there is an average tendency toward slightly higher outflow velocities at a given massloss rate for C-rich stars (cf., e.g., Olofsson 2004). This could be a natural consequence of all these winds being driven by carbon grains, but with an increasing amount of carbon being available for condensation, going from $\mathrm{O}$-rich to $\mathrm{C}$-rich objects.

\section{Conclusions}

We have investigated the viability of a scenario where winds of O-rich AGB stars are driven by small amounts of carbon grains. Our approach allows us to estimate both lower and upper limits for the required non-equilibrium effects in the gas, with one end of the spectrum being defined by the minimum amount of carbon dust necessary to drive an outflow and the other by the decreasing amounts of silicates being formed in the wind with increasing availability of carbon. Our model shows that significant amounts of forsterite grains (recently found in comets by STARDUST) can form in such winds.

The proposed scenario provides a natural explanation for the observed similarities in wind properties of O-rich and C-rich cool giants and implies a smooth transition for stars with increasing carbon abundance, from M-type to C-type AGB stars, probably solving the longstanding problem of the driving mechanism for stars with C/O close to one (S-type AGB stars; cf., e.g., Ramstedt et al. 2006).

Crucial, if non-trivial, tests of the scenario can be provided by observations that can assess the relative abundances of molecules in the critical dust formation region, in order to check for non-equilibrium effects in the gas phase. A direct detection of carbon grains in the spectra is rather unlikely: the optical properties of amorphous carbon lead to remarkably featureless IR spectra. On the other hand, $\mathrm{SiC}$, which is often used as an indicator for dust in C-rich stars due to its pronounced feature around $11 \mu \mathrm{m}$, will hardly be produced in sufficient quantities to be detected above the background of amorphous carbon and the nearby silicate feature. Considering the relative opacities of $\mathrm{SiC}$ and silicates, this would require at least as much $\mathrm{Si}$ to be bound in $\mathrm{SiC}$ as in silicates, which seems unlikely in an environment with $\mathrm{C} / \mathrm{O}<1$.

Acknowledgements. S.H. acknowledges support from the Swedish Research Council (Vetenskapsrådet). The Dark Cosmology Centre is funded by the Danish National Research Foundation.

\section{References}

Andersen, A. C., Höfner, S., \& Gautschy-Loidl, R. 2003, A\&A, 400, 981

Cherchneff, I. 2006, A\&A, 456, 1001

Gautschy-Loidl, R., Höfner, S., Jørgensen, U. G., \& Hron, J. 2004, A\&A, 422, 289

Höfner, S. 2007, in Why Galaxies Care About AGB Stars, ed. F. Kerschbaum, C. Charbonnel, \& R. Wing, ASP Conf. Ser., in press [arXiv: astro-ph/0702444]

Höfner, S., Gautschy-Loidl, R., Aringer, B., \& Jørgensen, U. G. 2003, A\&A, 399,589

Jäger, C., Mutschke, H., \& Henning, Th. 1998, A\&A, 332, 291

Jäger, C., Dorschner, J., Mutschke, H., et al. 2003, A\&A, 408, 193

Justtanont, K., Bergman, P., Larsson, B., et al. 2005, A\&A, 439, 627

Nowotny, W., Aringer, B., Höfner, S., et al. 2005, A\&A, 437, 273

Ohnaka, K., Bergeat, J., Driebe, T., et al. 2005, A\&A, 429, 1057

Olofsson, H. 2004, in Asymptotic Giant Branch Stars, ed. H. Habing, \& H. Olofsson (New York: Springer), 325

Ramstedt, S., Schöier, F. L., Olofsson, H., \& Lundgren, A. A. 2006, A\&A, 454, L103

Rouleau, F., \& Martin, P. G. 1991, ApJ, 377, 526

Schöier, F. L., Olofsson, H., \& Lundgren, A. A. 2006, A\&A, 454, 247

Weiner, J. 2004, ApJ, 611, L37

Woitke, P. 2006, A\&A, 460, L9

\footnotetext{
3 Note, again, that the degree of condensation of $\mathrm{Si}$ is a direct measure of the amount of silicate grains formed in the wind.
} 